\title{
OBSTRUCTIVE SLEEP APNEA - EPIDEMIOLOGY, CONSEQUENCIES AND PROSTHETIC REHABILITATION. A REVIEW
}

\author{
Sumit Katoch ${ }^{1}$, Manjit Kumar ${ }^{2}$, Amrit Khosla ${ }^{3}$, Ritu Batra ${ }^{4}$, Navjot Kaur ${ }^{5}$ \\ ${ }^{1}$ PG Student, Department of Prosthodontics, Bhojia Dental College and Hospital, Himachal Pradesh, India. \\ ${ }^{2}$ Professor and Head of Department, Department of Prosthodontics, Bhojia Dental College and Hospital. \\ ${ }^{3}$ Professor, Department of Prosthodontics, Bhojia Dental College and Hospital, Himachal Pradesh, India. \\ ${ }^{4}$ Reader, Department of Prosthodontics, Bhojia Dental College and Hospital, Himachal Pradesh, India \\ ${ }^{5}$ PG Student, Department of Prosthodontics, Bhojia Dental College and Hospital, Himachal Pradesh, India.
}

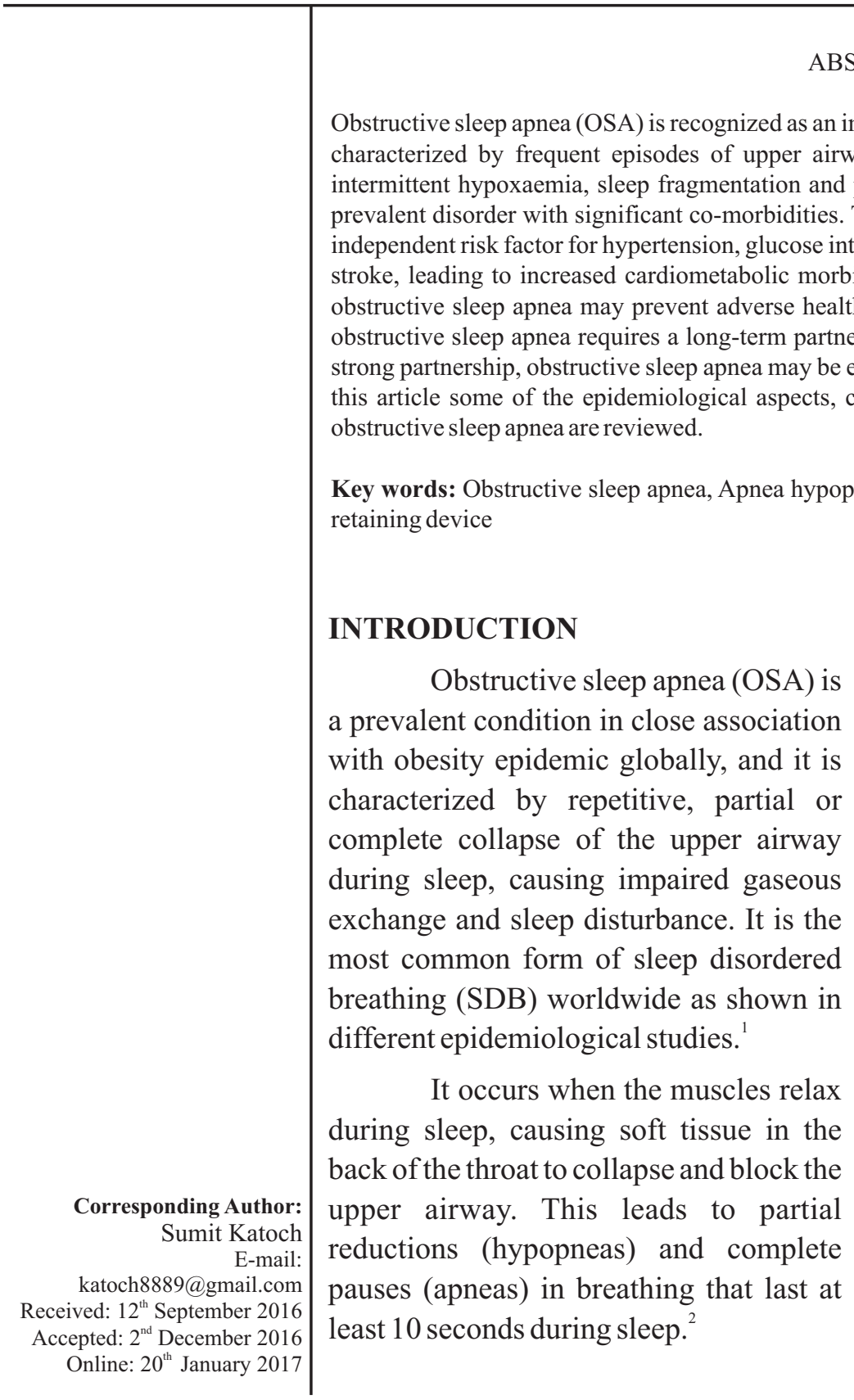

\section{ABSTRACT}

Obstructive sleep apnea (OSA) is recognized as an important health issue in the last two to three decades. It is obstructive sloep annea may prevent adreating the patient with obstructive sleep apnea requires a long-term partnership between patient and healthcare providers. With a this article some of the epidemiological aspects, consequences, medical and dental treatment options of Key words: Obstructive sleep apnea, Apnea hypopnea index, Mandibular repositioning appliance, Tongue retaining device

\section{INTRODUCTION}

a prevalent condition in close association with obesity epidemic globally, and it is characterized by repetitive, partial or complete collapse of the upper airway during sleep, causing impaired gaseous exchange and sleep disturbance. It is the most common form of sleep disordered breathing (SDB) worldwide as shown in different epidemiological studies.

during sleep, causing soft tissue in the back of the throat to collapse and block the upper airway. This leads to partial pauses (apneas) in breathing that last at least 10 seconds during sleep. ${ }^{2}$
A common measurement of sleep apnea is the apnea-hypopnea index (AHI). This is an average that represents the combined number of apneas and hypopneas that occur per hour of sleep. ${ }^{2}$

Obstructive sleep apnea (OSA) impacts negatively the health and well being of millions of peoples worldwide. It is an independent risk factor for hypertension, heart failure, myocardial infarction, stroke and arrhythmias, where severity of sleep apnea typically predicts risk. OSA may also impair cognitive function, mood, diabetic control, and potentially liver and renal function. ${ }^{3}$

Treatment of OSA requires extensive communication and strong collaboration between physician and 
patient to develop the most effective individual treatment plan. The first two steps in managing an individual patient with OSA are to decide what to treat and then how to treat. Close collaborative follow up is required to determine how effectively the treatment plan is working. Large-scale studies focusing on individuals with mild to moderate disease can provide the much needed insight into how best to manage the majority of individuals with mild-moderate OSA. ${ }^{3}$

\section{PREVALENCE}

- OSA can occur in any age group, but prevalence increases between middle and older age. OSA with resulting daytime sleepiness occurs in at least four percent of men and two percent of women. About 24 percent of men and nine percent of women have the breathing symptoms of OSA with or without daytime sleepiness. About 80 percent to 90 percent of adults with OSA remain undiagnosed.

- OSA occurs in about two percent of children and is most common at preschool ages. ${ }^{3}$

\section{Indications for oral appliance therapy}

- Patients with mild to moderate obstructive sleep apnea

- Patients who are unable to tolerate CPAP

- Sufficient number of healthy teeth

- Absence of any periapical pathology

- Absence of any severe respiratory disorder

- Well formed ridges in completely edentulous patients

\section{Contraindications}

- Severe obstructive sleep apnea

- Severe respiratory disorders

- Medically compromised patients

- Periodontally compromised teeth

- Psychological problems

\section{TYPES}

- Mild OSA:AHI of 5-15

Involuntary sleepiness during activities that require little attention, such as watching TV or reading.

- Moderate OSA: AHI of 15-30

Involuntary sleepiness during activities that require some attention, such as meetings or presentations.

- Severe OSA: AHI of more than 30

Involuntry sleepiness during activities that require more active attention, such as talking or driving. ${ }^{2}$

\section{SIGNS \& SYMPTOMS}

- Fluctuating oxygen levels, Increased heart rate, Chronic elevation in daytime blood pressure

- Increased risk of stroke, Higher rate of death due to heart disease, Impaired glucose tolerance and insulin resistance, Impaired concentration, Mood changes, Increased risk of being involved in a deadly motor vehicle accident, Disturbed sleep of the bed partner. ${ }^{3}$

\section{RISK GROUPS}

The major risk factors for OSA include advanced age, male sex and obesity, although the underlying mechanisms remain unclear. ${ }^{4}$

Age: The increased prevalence of SDB in the elderly appears to plateau after $65 \mathrm{yr}$, it is estimated to be 10 per cent. ${ }^{5}$ However, when the prevalence is controlled for body mass index, the severity appears to decrease with age. ${ }^{6}$ Mechanisms proposed for the increased prevalence of sleep apnea in the elderly include increased deposition of fat in the parapharyngeal area, lengthening of the soft palate, and changes in body structures surrounding the pharynx.

Sex: It is not clear why OSA is more common in men than women. It can be attributed to anatomical and functional properties of the upper airway and in the ventilatory response to the arousals from sleep ${ }^{8}$. Imaging studies have revealed that men have increased fat deposition around pharyngeal airway as compared with women. ${ }^{9}$

Obesity: Obesity / visceral obesity is the major risk factor for the development of OSA, it is thought to be associated with anatomic alterations that predispose to upper airway obstruction during sleep, by increasing adiposity around the pharynx and body. Central obesity has been associated with reduction in lung volume, which leads to a loss of caudal traction on the upper airway, and hence, an increase in pharyngeal collapsibility. ${ }^{10}$ 
Family history and genetic predisposition: Familial aggregation and genetics factors are thought to play a role in the development of OSA. First degree relatives of those with OSA increases the relative risk compared to those without OSA by 1.5-2.0, and familial susceptibility to OSA increases directly with the number of affected relatives. ${ }^{11}$

Craniofacial abnormalities: Differences in craniofacial morphology may explain some of the variation in risk for OSA in different ethnic groups. Previous studies have shown that craniofacial abnormalities are important in the pathogenesis of OSA, particularly in non obese patients. ${ }^{12}$

Smoking and alcohol consumption: Cigarette smoking and alcohol have been shown to be risk factors for OSA. Smoking is associated with a higher prevalence of snoring and sleep-disordered breathing. Alcohol relaxes upper airway dilator muscles, increases upper airway resistance and may induce OSA in susceptible therefore, alcohol intake can prolong apnea duration, suppress arousals, increase frequency of occlusive episodes and worsen the severity of hypoxaemia. ${ }^{13}$

\section{DIAGNOSIS}

Sleep apnea must first be diagnosed at a sleep center or lab during an overnight sleep study, or "polysomnogram." The sleep study charts vital signs such as brain waves, heart beat and breathing.

\section{TREATMENT}

- Continuous positive airway pressure (CPAP) CPAP is the standard treatment option for moderate to severe cases of OSA and a good option for mild sleep apnea. This was introduced for the treatment of sleep apnea in 1981, it provides a steady stream of pressurized air to patients through a mask that they wear during sleep. This airflow keeps the airway open, preventing pauses in breathing and restoring normal oxygen levels. ${ }^{2}$

\section{- Oral appliances}

An oral appliance is an effective treatment option for people with mild to moderate OSA who either prefer it to CPAP or are unable to successfully comply with CPAP therapy.
Oral appliances are indicated for use in patients with primary snoring, mild and moderate OSA cases who do not respond or are not appropriate candidates for treatment with behavioral measures such as weight loss or sleep position change. It can also be considered in patients who are not amenable to CPAP therapy or surgery. A review of oral appliance therapy for OSA in 1995 signaled the entry of dentistry into the field of mainstream sleep medicine. Adjustable mandible advancing oral appliances became the predominant form of dental therapy for sleep disordered breathing in 1990's.

\section{Mechanisms of Action of Oral Appliances}

Oral appliances may improve upper airway patency during sleep by enlarging the upper airway and/or by decreasing upper airway collapsibility (e.g., improving upper airway muscle tone). ${ }^{1415,16}$

\section{Types of Oral appliances}

Two types:

\section{1) Appliances for dentulous patient: \\ - Tongue Retaining Device ( Figure.1 ) \\ - Mandibular Advancing type :}

a) Non- Titratable Type : The mandibular advancing and non-titratable type of oral appliance is a custom-made monoblock device connected by two separate arches (maxillary and mandibular) without an advancing mechanism. Examples are given below.

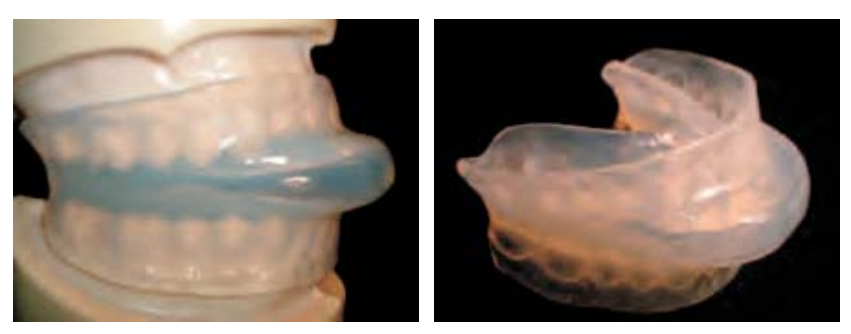

Figure1: Tongue Retaining Device

- Esmarch appliance ( Figure 2)

- Nocturnal airway patency appliance(NAPA) (Figure 3)

- Mandibular repositioner (Figure 4)

- Snore guard (Figure 5)

b) Titratable Type : The mandibular advancing and 

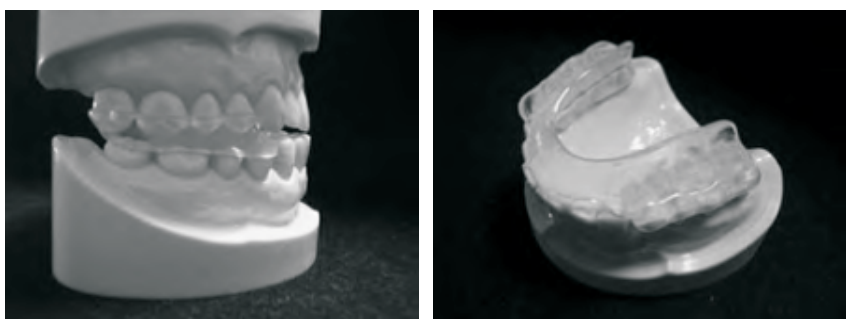

Figure 2: Esmarch appliance
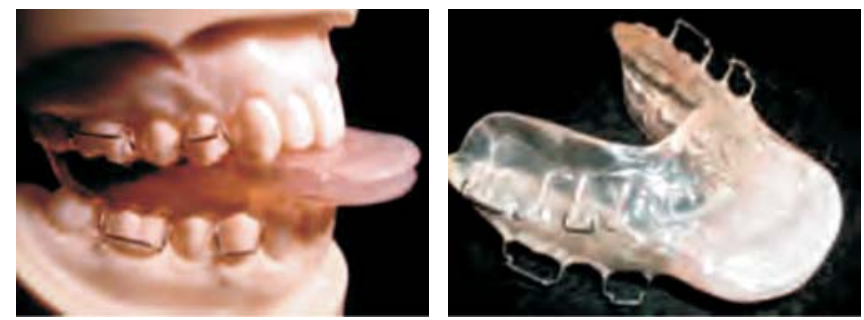

Figure 3: Nocturnal airway patency appliance (NAPA)
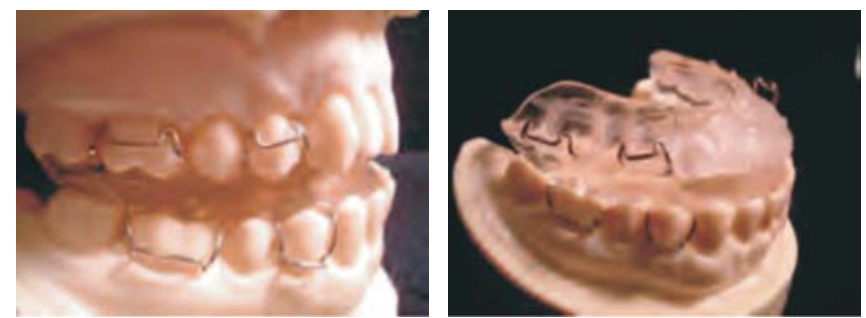

Figure 4: Mandibular repositioner
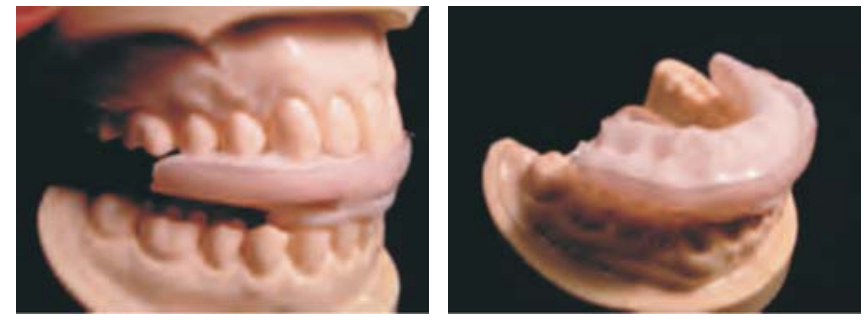

Figure 5: Snore guard

titratable type of oral appliance is a custom- made twopiece appliance composed of two separate arches (maxillary and mandibular) containing an advancing mechanism. Examples are given below.

- Klearway (Figure 6): This custom-made two-piece appliance is composed of two separate arches (maxillary and mandibular) to advance the mandible with a screw $(0.25 \mathrm{~mm} / 1$ turn $)$ to determine the ideal forward position of the mandible required to adequately open the airway. A total of 44 forward positions are available in increments of $0.25 \mathrm{~mm}$, which covers a full $11.0 \mathrm{~mm}$ range of anterior-posterior movement.
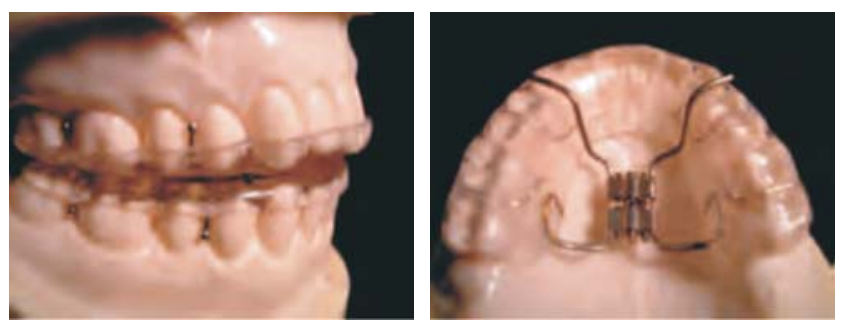

Figure 6: Klearway

- Thornton adjustable positioner (TAP)( Figure 7 ) : This appliance is composed of two separate arches (maxillary and mandibular) containing an advancing mechanism and a base and hook assembly with an internal adjustment mechanism.

- Herbst (Figure 8) : This device contains an advancing mechanism which has two sets of pistons and tubes to keep the mandible in the forward position required to adequately open the airway.
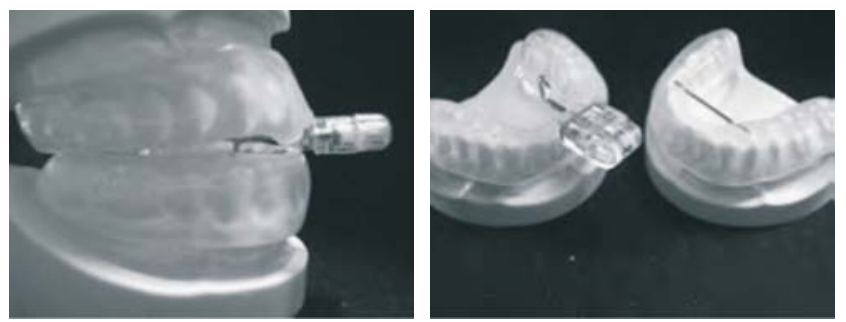

Figure.7: Thornton adjustable positioner (TAP)
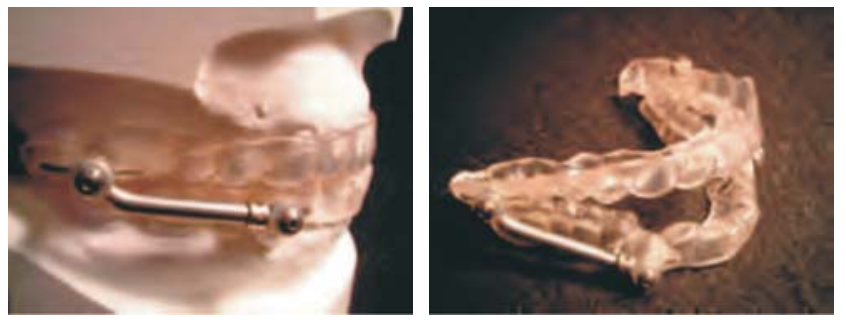

Figure 8: Herbst

\section{2) Appliances for edentulous patient:}

- Oral appliances for edentulous patient (Figure 9)

- Implant reatained oral appliance (Figure 10)

Technique of fabrication of oral appliance for edentulous patient :

1. Make record bases on the master casts and place them clinically to evaluate retention and stability.

2. Establish a vertical and protrusive jaw position 5 to $8 \mathrm{~mm}$ open and anterior to the physiologic rest 


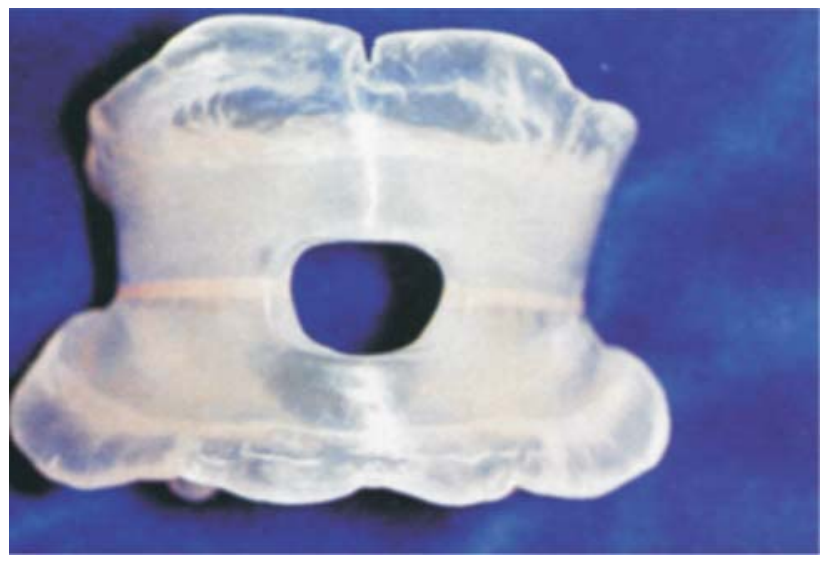

Figure 9: Oral appliance for edentulous patient
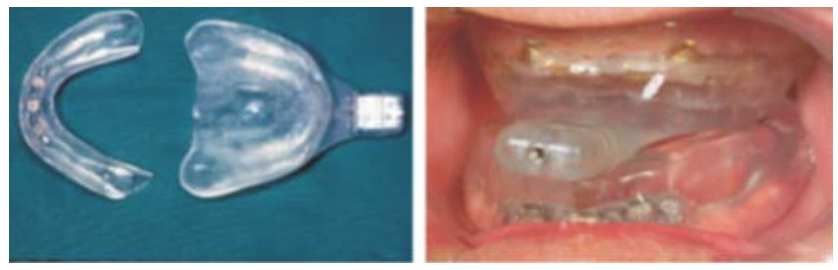

Figure 10: Implant retained oral appliance for edentulous patient

position. Allow sufficient time for the patient to accommodate to this position. The patient should not strain to maintain this posture and should show no symptoms of muscle temporomandibular joint syndrome.

3. Modify the record bases until uniform contact is achieved in both the most retruded position and the protrusive position at the increased vertical dimension.

4. Embed radiopaque string into the labial surfaces of the wax occlusion rims to permit radiographic analysis (Figure 11).

5. Make cephalograms (1) at the physiologic rest position, (2) with the record bases at the most

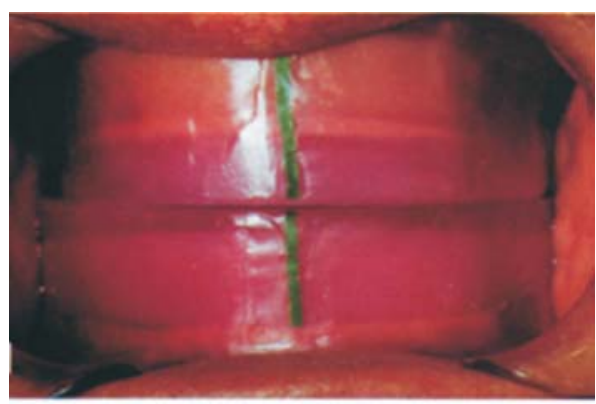

Figure.11- Radiopaque string in place for radiographic analysis retruded mandibular position, and (3) at the protrusive position. The cephalograms may reveal variations in linear space between the base of the tongue and the posterior pharyngeal wall. An increase in airway space from the most retruded mandibular position to the protrusive position may be significant (Figure 12).

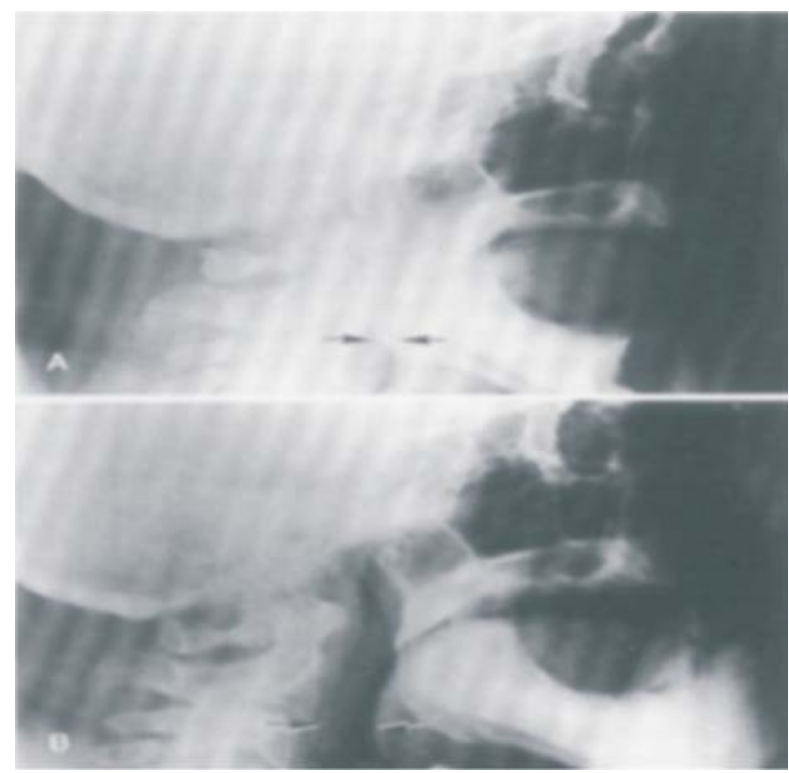

Figure.12-Cephalogram showing significant changes in space in retruded and protruded mandibular position

6. Make an interocclusal jaw relation record at the protrusive position and articulate the casts in a semiadjustable articulator (Figure 13).

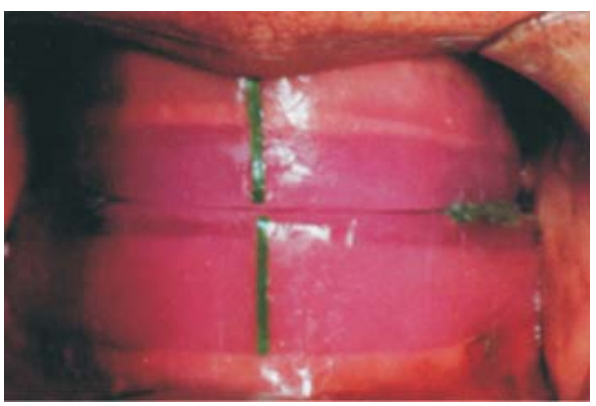

Figure 13: Interocclusal jaw relation record is made at established protruded position

7. Leave a 1-2 $\mathrm{mm}$ space between maxillary and mandibular occlusal surfaces when waxing is completed. This space will allow for placement of acrylic resin on the opposing members of the prosthesis after processing. The buccal and lingual 
contours of the maxillary and mandibular wax patterns should be continuous. An orifice is created anteriorly to assist with ventilation (Figure 14,15).

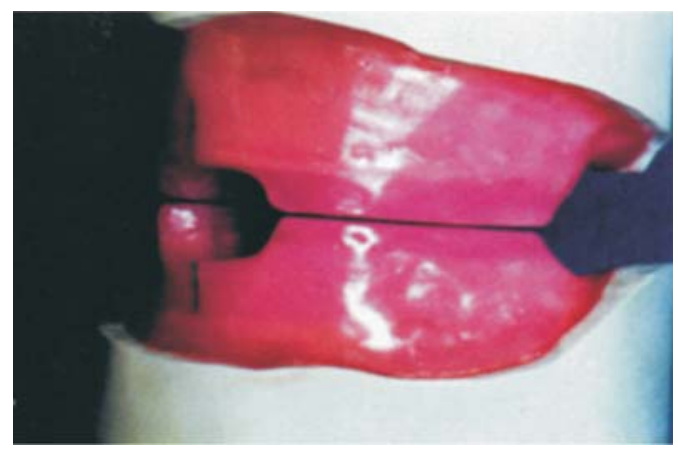

Figure14: Separation between maxillary and mandibular wax patterns

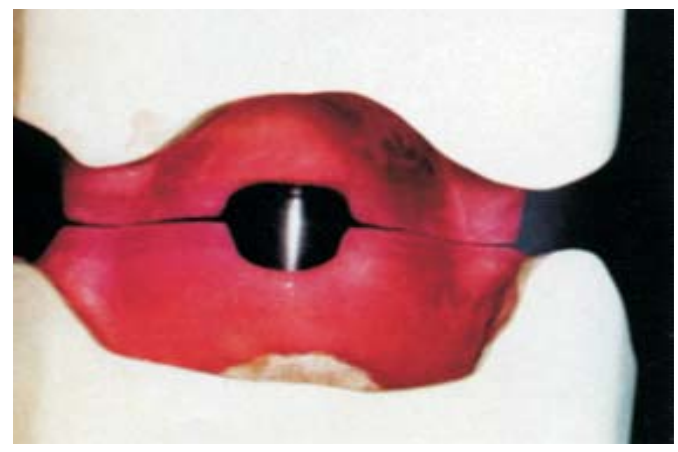

Figure.15- Continuity on buccal and lingual aspects

8. Seal the wax patterns and record bases to their respective casts. Invest and process in heat cure clear acrylic resin. Before separation from the master casts, remount in the articulator. The incisal guide pin of the articulator should contact the guide table and a continuous space should exist or be provided between the maxillary and mandibular components. Polish the cameo surfaces of both components before acrylic resin is used to join the opposing members ( Figure 16).

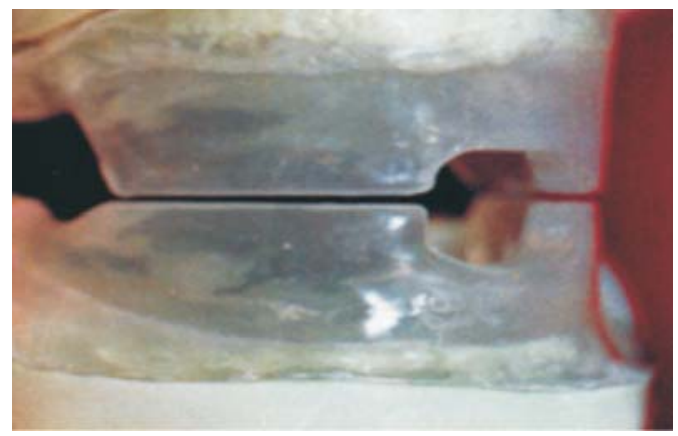

Figure.16- Processed portions of prosthesis are returned to articulator
9. Use a visible light curing resin to complete the union between the maxillary and mandibular segments. Apply bonding agent to the opposing surfaces before the light-cured resin is adapted. While it is in a moldable state, place the resin on the opposing surfaces and close the articulator to the established vertical position. Remove excess resin before using a light source to cure the material. Direct the light source to both the buccal and the lingual surfaces to cure the material sufficiently before its separation from the articulator (Figure 17).

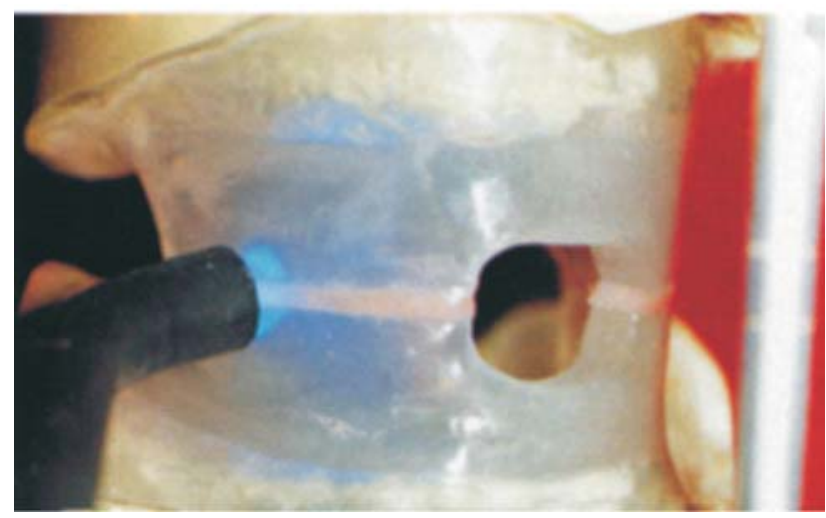

Figure 17: Light curing of prosthesis

10. Separate the prosthesis from the casts, place it in the Triad unit for final curing, and finish it (Figure 18).

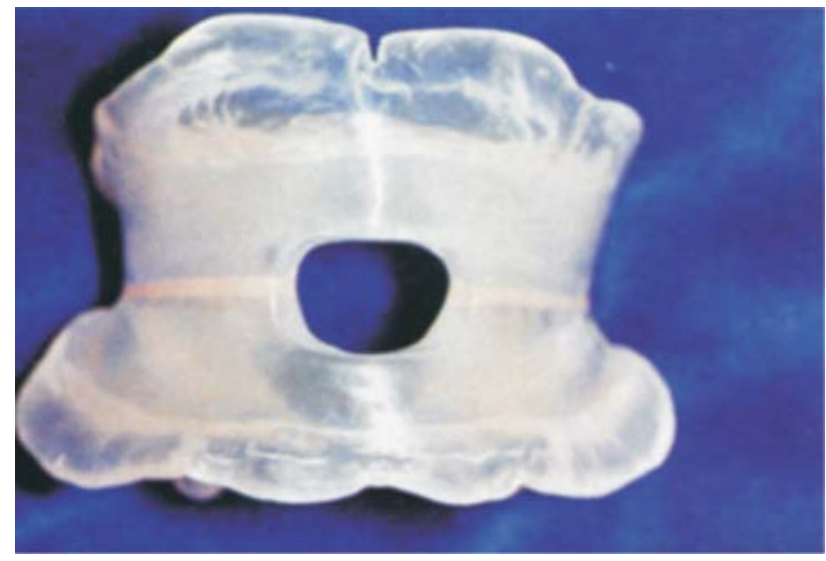

Figure18: Completed prosthesis

11. Use pressure-indicating paste clinically to reveal possible sources of tissue irritation and adjust the positions as needed. Instruct the patient on how to 
insert the prosthesis properly and how to remove it. Provide counseling on procedures for care of the prosthesis at home. Complete dentures should be removed for a specific period during the day to avoid or minimize the adverse effects of continuous wear (Figure 19).

12. During the follow up evaluation assess the patient's sleep and check for the onset of hypersomnolence and other related symptoms. The prosthesis should remain stable and retentive throughout the night, and the patient should be free of myofacial or temporomandibular joint symptoms. ${ }^{15}$

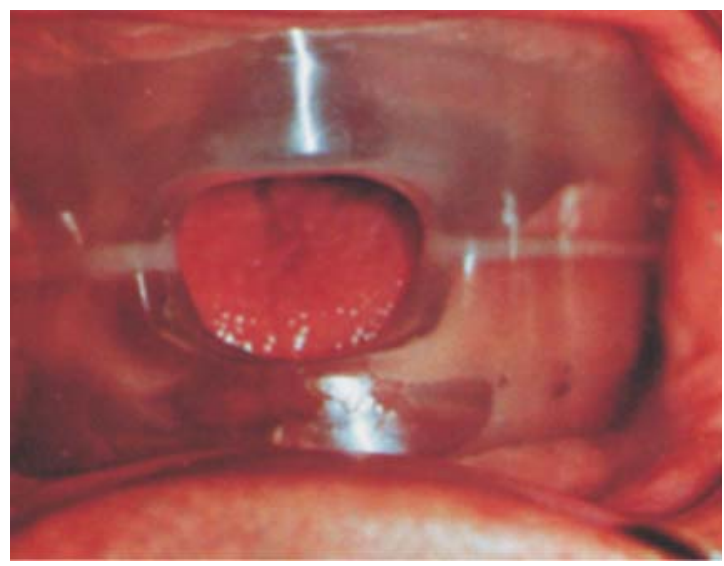

Figure 19: Completed prosthesis

\section{Surgery}

Surgery is a treatment option for OSA when noninvasive treatments such as CPAP or oral appliances have been unsuccessful. It is most effective when there is an obvious anatomic deformity that can be corrected to alleviate the breathing problem. One of the most common surgical methods is uvulopalatopharyngoplasty (UPPP), which trims the size of the soft palate and may involve the removal of the tonsils and uvula. Adenotonsillectomy, the surgical removal of the tonsils and adenoids, is the most common treatment option for children with OSA. Other children with sleep apnea may benefit from CPAP.

\section{Behavioral changes}

Weight loss benefits many people with sleep apnea, and changing from back-sleeping to sidesleeping may help those with mild cases of OSA.

\section{Position Therapy}

A treatment used for patients suffering from mild OSA. Patients are advised to stay off of the back while sleeping and raise the head of the bed to reduce symptoms.

\section{SUMMARY}

Most of the snoring and OSA patients can be successfully treated using surgical procedures, NCPAP, behavioral modification, and/or dental devices. However, because obstruction may occur at differing levels of the upper airway, any specific treatment modality will not address the problems of all patients. Marklund et found MADs satisfactorily treated patients with mild and moderate sleep apnea but had poor results with those classified as severe. They have suggested that oral devices may not address larger obstructions in the upper airway, perhaps extending to the epiglottis, and this may account for their decreased success in these patients. ${ }^{17}$ Powell et al believe this may also account for the low success rate in treatment with UPPP. ${ }^{18}$ Recently, dental participation in management of the patients through oral prosthesis therapy has been accepted as an appropriate treatment modality by the American Sleep Disorders Association. Indication for oral devices are those patients with primary snoring or mild OSA who do not respond to or are not appropriate candidates for treatment with behavioral measures, those with moderate-to-severe OSA who are intolerant of or refuse treatment with nasal CPAP, and those who refuse or are not candidates for surgical treatment.

\section{REFERENCES}

1. Jamie C.M. Lam, S.K Sharma \& Bing Lam. Obstructive sleep apnea: Definition, epidemiology and natural history. Indian J Med Res 131, February 2010,pp 165-170

2. Obstructive Sleep Apnea(American Academy of Sleep Medicine )

3. Sigrid Veasey. Treatment of obstructive sleep apnea. Indian J Med Res 131, February 2010, pp 236-244.

4. Eckert DJ, Malhotra A. Pathophysiology of adult obstructive sleep apnea. Proc Am Thorac Soc 2008; 5 : 144-53.

5. Young T, Skatrud J. Peppard PE. Risk factors for obstructive sleep apnea in adults. JAMA 2004; 291 : 2013-6.

6. Young T, Palta M, Dempsey J, Skatrud J, Weber S, Badr S. The occurrence of sleep-disordered breathing among middleaged 
adults. N Engl J Med 1993; $328: 1230-5$.

7. Eikermann $\mathrm{M}$, Jordan AS, Chamberlin NL, Gautam S, Wellman A, Lo YL. The influence of aging on pharyngeal collapsibility during sleep. Chest 2007; 131: 1702-9.

8. Jordan AS, McEvoy RD. Gender differences in sleep apnea: epidemiology, clinical presentation and pathogenic mechanisms. Sleep Med Rev 2003; 7 : 377-89.

9. Whittle AT, Marshall I, Mortimore IL, Wraith PK, ellar RJ, Douglas NJ. Neck soft tissue and fat distribution: comparison between normal men and women by magnetic resonance imaging. Thorax 1999; 54:323-8.

10. Schwartz AR, Patil SP, Laffan AM, Polotsky V, Schneider H, Smith PL. Obesity an obstructive sleep apnea - pathogenic mechanisms and therapeutic approaches. Proc Am Thorac Soc $2008 ; 5: 185-92$.

11. Lam B, Lam DCL, Ip MSM. Obstructive sleep apnea in Asia. Int J Tuberc Lung Dis 2007: 11: 2-11.

12. Khoo SM, Tan WC, Ng TP, Ho CH. Risk factors associated with habitual snoring and sleep-disordered breathing in a multi-ethnic Asian population: a population-based study. Resp Med 2004; 98 : 557-66.
13. Mitler MM, Dawson A, Henriksen SJ, Sobers M, Bloom FE. Bedtime ethanol increases resistance of upper airways and produces sleep apneas in asymptomatic snorers. Alcohol Clin Exp Res 1998; $12: 801-5$.

14. Lt Col B Jayan, Brig BNBM Prasad, SM, VSM, Col RK Dhiman. Role of Oral Appliances in the Management of Sleep Disorders. MJAFI 2009; $65: 123-127$.

15. Jack B. Meyer, Jr., D.M.D.,and Rodney C. Knudson, D.M.D., M.S. Fabrication of a prosthesis to prevent sleep apnea in edentulous patients.

16. Aarnoud Hoekema, Frist de vries, Kees Heydenrijk. Implantretained oral appliances : a novel treatment for edentulous patients with obstructive sleep apnea - hypoapnea syndrome. Clin.Oral Impl. Res. 18, 2007/383-387.

17. Marklund M, Franklin KA, Sahlin C, Lundgren R.. The effect of a mandibular advancement device on apneas and sleep in patients with obstructive sleep apnea. Chest 1998;113:707-13.

18. Powell NB, Riley RW, Robinson A. Surgical management of obstructive sleep apnea syndrome. Clin Chest Med 1998; 19:77-86. 